a protein adduct that was $41 \mathrm{Da}$ lower in molecular weight than that expected for the parent ML210 compound. Furthermore, an alkyne probe analog of ML210 enriched GPX4 from cancer cells and revealed that this compound had fewer off targets than $\alpha$-chloroacetamide inhibitors of GPX4.

Surprisingly, ML210 did not form covalent adducts with purified GPX4, leading Eaton et al. ${ }^{1}$ to hypothesize that the compound acts as a cloaked electrophilic prodrug requiring metabolic activation in cells. The chemical transformation that ML210 undergoes in cells was deduced by a remarkably clever series of experiments involving iterative chemical-synthesisdriven explorations of the structure-activity relationship of ML210 analogs. A key clue was the requirement of the nitroisoxazole ring for activity, a chemical group that can undergo ring opening to an $\alpha$-nitroketoxime under basic conditions ${ }^{8}$. JKE-1674, an analog of ML210 in which the nitroisoxazole ring was replaced with an $\alpha$-nitroketoxime, was synthesized and found to induce ferroptosis and covalently label GPX4 in cells. However, the investigative work of the authors was not done yet, as JKE-1674 also did not react with purified GPX4, indicating that a further chemical transformation occurred in cells to generate the relevant bioactive electrophile.

Subsequent mechanistic studies revealed that the $\alpha$-nitroketoxime group of JKE-1674 can be converted into a nitrile oxide JKE1777 (Fig. 1b), which proved capable of reacting with purified GPX4. The molecular weight of the adduct formed by the reaction of JKE-1777 with the selenocysteine of GPX4 was identical to that of the adduct observed for ML210 in cells. Additional analogs of ML210-containing substituents, such as a nitrolic acid, that spontaneously generate nitrile oxides displayed similar activity to that of ML210, providing further evidence that a nitrile oxide electrophile is responsible for the GPX4 inhibitory and ferroptosis-inducing activity of ML210.

The enhanced selectivity and stability of compounds such as ML210 and JKE-1674 enabled experiments that were not possible with original $\alpha$-chloroacetamide GPX4 inhibitors. For instance, a CRISPR-based suppressor screen using ML210 identified genes essential for ferroptosis that were not previously discovered in screens with other ferroptosis-inducing compounds. Additionally, as a hint of experiments to come, Eaton et al. ${ }^{1}$ showed that mice could be orally dosed with JKE-1674, and the compound could be detected in serum for up to $24 \mathrm{~h}$.

The precise mechanism by which the nitroisoxazole group of ML210 is converted into a reactive nitrile oxide in a cellular context remains unknown. Identifying the conditions or enzymes, if any, responsible for this conversion may represent a promising avenue of future research. In particular, if enzymatic pathways with greater expression in cancer cells can be leveraged to generate nitrile oxide inhibitors of GPX4 from prodrug precursors, this could allow more selective induction of ferroptosis, which remains a persistent translational concern, given that GPX4 inactivation has the potential to produce toxic effects in organs such as the kidney9.

We also point out that one of the original clues motivating Eaton et al. ${ }^{1}$ to investigate ML210 was its activity in a screen of $>800$ human cancer cell lines in which the compound produced a pattern of cell killing that closely resembled those of other GPX4 inhibitors (http://portals.broadinstitute. org/ctrp.v2.1). This finding, and others ${ }^{10}$, underscores the power of large-scale, cellbased screening for illuminating unexpected mechanistic relationships across structurally diverse compound classes. Although the molecular evidence for these hidden connectivities may only be uncovered by diving into the complex context of the human cell, Eaton et al. ${ }^{1}$ demonstrate how the analytical tools available to the modern chemical detective can make such an ambitious mystery tour well worth taking. Finally, we wonder whether further investigation of nitroisoxazoles and $\alpha$-nitroketoximes as masked electrophiles may reveal the potential to develop selective ligands of other (seleno)cysteine proteins, thereby further expanding the scope of covalent chemistry for probing the human proteome.

\section{Stefan G. Kathman and \\ Benjamin F. Cravatt (DD \\ Department of Chemistry, The Scripps Research \\ Institute, La Jolla, CA, USA. \\ e-mail:skathman@scripps.edu; cravatt@scripps.edu}

Published online: 30 March 2020

https://doi.org/10.1038/s41589-020-0511-3

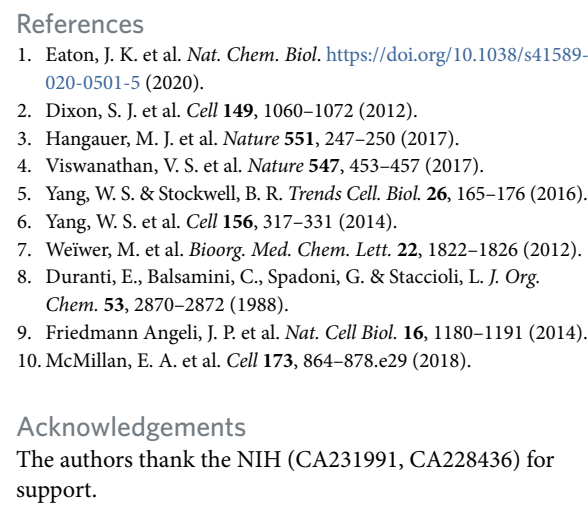

Competing interests

The authors declare no competing interests.

\title{
Anti-HIV agents inspired by antibodies
}

Emergence of drug resistance limits the efficacy of HIV drugs, which currently requires life-long administration. In vitro high-throughput screening for competition with a broadly neutralizing antibody of HIV identified a small molecule that extends the strategies for targeting HIV.

\section{Shan Su and Shibo Jiang}

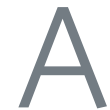

IDS-related mortality has declined considerably because of the widespread application of combination antiretroviral therapy. However, pretreatment resistance to antiretroviral drugs has been reported in many countries ${ }^{1}$. Therefore, development of anti-HIV drugs against new targets and with high genetic barriers to resistance is urgently needed. Xiao et al. ${ }^{2}$ now describe a new class of small-molecule HIV-1 inhibitors targeting the highly conserved membrane-proximal external region (MPER) in the HIV-1 enveloped glycoprotein Env (Fig. 1), which contains 
a

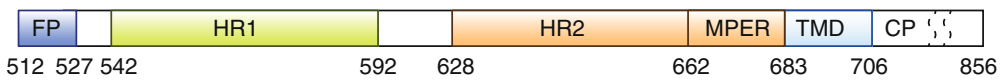

b

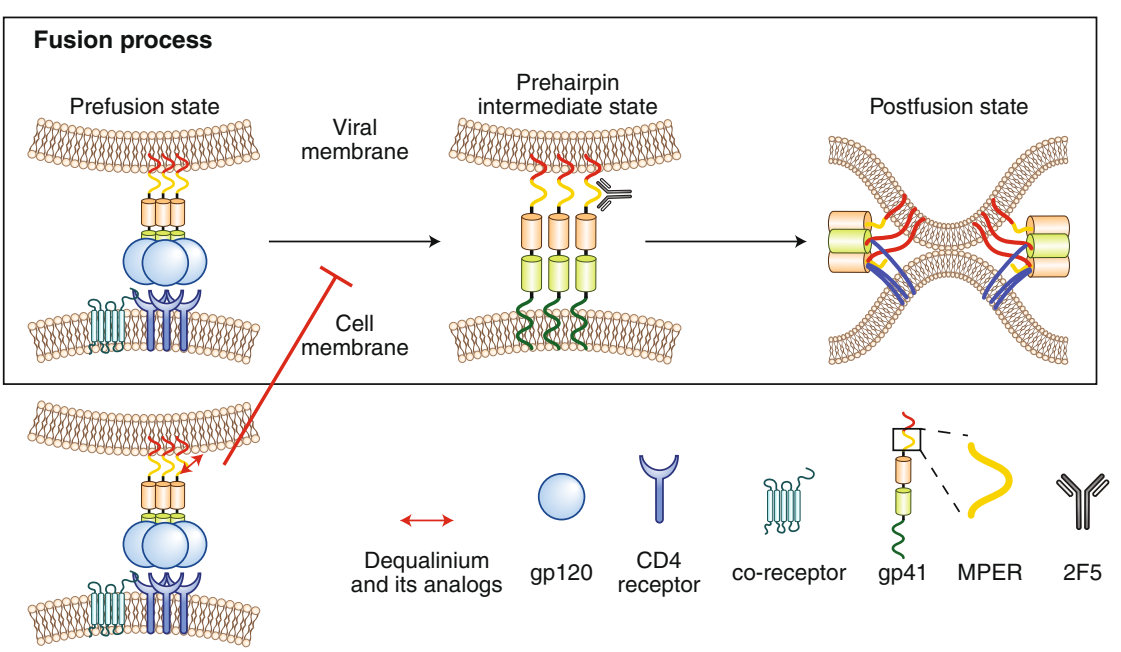

Fig. 1 | The functional domains in the HIV-1 Env, the paradigm of HIV entry and the inhibition mechanism of dequalinium and its analogs on HIV infection. a, Schematic diagram of HIV-1 Env. The positions of the gp120, the fusion peptide (FP), the heptad repeats 1 and 2 (HR1 and HR2, respectively), the membrane-proximal external region (MPER), the transmembrane domain (TMD) and the cytoplasmic region (CP) are shown. b. Dequalinium and its analogs binds to the MPER region at the prefusion stage, and this binding halts the conformational change of HIV Env into the prehairpin intermediate and postfusion conformations, thus inhibiting HIV-1 infection.

the epitopes recognized by several broadly neutralizing antibodies (bNAbs). MPER is a region of glycoprotein 41 (gp41) adjacent to the viral membrane and is required for viral infectivity ${ }^{3}$. Even though the exact role of MPER in the mechanism of viral fusion is still unknown, the bNAbs targeting MPER appear to block HIV-1 infection by binding to the prehairpin intermediate state of gp41. Therefore, the authors reasoned that small molecules targeting MPER could act similarly to the bNAbs. So far, the US Food and Drug Administration (FDA)-approved HIV small-molecule drugs mainly fall into four categories: nucleoside/nonnucleoside reverse-transcriptase inhibitors (NRTIs/ NNRTIs), protease inhibitors (PIs), integrase inhibitors (IIs), and entry inhibitors (EIs, including a fusion inhibitor and a co-receptor antagonist). Most drugs target either an enzyme active site (such as those of NRTIs/NNRTIs, PIs, and IIs) or a ligandbinding site (such as those of EIs). Also, several bNAbs have been isolated from chronically HIV-infected patients. Among them, the MPER-specific bNAbs, including 2F5 and 10E8, are highly potent and broadly effective against divergent HIV-1 strains ${ }^{4}$.

To investigate whether small-molecule compounds can mimic these bNAbs to bind the MPER, the authors performed an in vitro high-throughput screening assay seeking compounds that compete with 2F5 for binding MPER. After screening of a library of 162,106 compounds, dequalinium, an FDA-approved antimicrobial drug emerged. Dequalinium could bind MPER and effectively inhibit HIV-1 Env-mediated membrane fusion and HIV-1 infection in vitro. Structure-activity relationship analysis of dequalinium derivatives identified S2C3, which has improved potency against diverse HIV-1 strains. Mechanistic and NMR structural studies showed that $\mathrm{S} 2 \mathrm{C} 3$ bound to the conserved hydrophobic pockets formed by two adjacent MPERs at the prefusion stage of the viral life cycle, and S2C3 binding halted the conformational change of HIV-1 Env into the prehairpin intermediate and postfusion conformation, thus explaining its ability to inhibit HIV-1 infection (Fig. 1).

Use of a small-molecule drug can help overcome several of the disadvantages of an antibody-based drug, such as high cost of production, cold-chain transportation, and requirement for delivery by injection. Several series of small-molecule EIs have been identified previously on the basis of their ability to bind the corresponding target sites in the HIV-1 Env 5 . As a smallmolecule EI, S2C3 targets a site in the
HIV-1 Env distinct from those targeted by small-molecule EIs that have been identified so far. The new approach for the identification of $\mathrm{S} 2 \mathrm{C} 3$ based on its competitive inhibition of the MPER binding by the antibodies that have been proven to be effective in inhibiting HIV-1 infection is more straightforward and time saving, as it exploits the immune system, which has used evolution to find a feasible target.

Because anti-MPER bNAbs are effective against a broad range of HIV-1 strains, MPER remains one of the most desirable targets in the HIV-1 vaccine research field. As S2C3 did not interfere with binding of Env to the anti-gp120 antibody VRC01, it can be expected to have a synergistic effect with other EIs. Besides the potential as a direct drug candidate, S2C3 could stabilize HIV-1 Env in the prefusion conformation, making it potentially useful in a vaccine preparation to reduce the conformational flexibility of Env.

Finally, this antibody-based screening strategy for drug discovery could be applicable to many other human diseases, especially those caused by infectious agents. For example, Ebola virus is still epidemic in Africa, especially those poor tropic or subtropic areas. However, most of the clinical trial of Ebola virus disease therapeutics are based on antibodies, such as MAb114 and ZMapp ${ }^{6}$. This antibody-based approach will possibly facilitate the discovery of smallmolecule drugs for treatment of Ebola virus disease or COVID-19 caused by 2019-nCoV (also known as HCoV-19 or SARS-CoV-2) ${ }^{7}$, whereas small-molecule drugs are generally orally available and cost-effective compared to antibody drugs.

\section{Shan Su (D) ${ }^{1}$ and Shibo Jiang (D) 1,2四}

${ }^{1}$ Key Laboratory of Medical Molecular Virology (MOE/MOH/CAM), School of Basic Medical Sciences, Fudan University, Xuhui District, Shanghai, China. ${ }^{2}$ Lindsley F. Kimball Research Institute, New York Blood Center, New York, NY, USA.

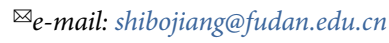

Published online: 22 April 2020 https://doi.org/10.1038/s41589-020-0521-1

\footnotetext{
References

1. Phillips, A. N. et al. Lancet HIV 1, e85-e93 (2014).

2. Xiao, T. et al. Nat. Chem. Biol. https://doi.org/10.1038/s41589020-0496-y (2020)

3. Salzwedel, K., West, J. T. \& Hunter, E. J. Virol. 73, 2469-2480 (1999).

4. Huang, J. et al. Nature 491, 406-412 (2012).

5. Lu, L., Yu, F., Cai, L., Debnath, A. K. \& Jiang, S. Curr. Top. Med. Chem. 16, 1074-1090 (2016).

6. Mulangu, S. et al. N. Engl. J. Med. 381, 2293-2303 (2019).

7. Jiang, S. et al. Lancet https://doi.org/10.1016/S0140-

6736(20)30419-0 (2020).

The authors declare no competing interests.
} 